\title{
2D Anisotropic Photonic Crystals of Hollow Semiconductor Nanorod with Liquid Crystals
}

\author{
Filiz Karaomerlioglu ${ }^{1, a}$, Sevket Simsek ${ }^{2, b}$, Amirullah M. Mamedov, \\ Ekmel Ozbay $2, d$ \\ ${ }^{1}$ Department of Electronic and Computer Education, Mersin University, Mersin, Turkey \\ ${ }^{2}$ Nanotechnology Research Center (NANOTAM), Bilkent University 06800, Ankara, Turkey \\ afilizkrm@mersin.edu.tr, ${ }^{b}$ sevkets@bilkent.edu.tr, ${ }^{c}$ mamedov@bilkent.edu.tr, ${ }^{d}$ ozbay@bilkent.edu.tr
}

Keywords: Anisotropic Photonic Crystal, Semiconductor, Liquid Crystal

\begin{abstract}
Photonic crystals (PCs) have many applications in order to control light-wave propagation. A novel type of two-dimensional anisotropic PC is investigated band gap and optical properties as a hollow semiconductor nanorod with nematicliquid crystals (LC). The PC structure composed of an anisotropic nematicLC in semiconductor square hollow nanorod is designed using the plane wave expansion (PWE) method and finite-difference time-domain (FDTD) method. It has been used 5CB (4-pentyl-4 -cyanobiphenyl) as LC core, and Tellurium (Te) as square hollow nanorod material.The PC with hollow Tenanorod with nematicLC is compared with the PC with solid Tenanorodand the PC with hollow Tenanorod.
\end{abstract}

\section{Introduction}

Progress in solid-state physics, optics of spatially structures, and nanotechnologies based on a variety of the physical and chemical processes has strongly stimulated and motivated the investigation into the properties of photonic crystals and resultedin the growth of applications of photonic bandgap materials, i.e. artificially structured materials where optical parameters are periodically modulated in space with a period of a unit photonic crystal cell on the order of the optical wavelength.Previous studies about photonic band-gap structures (PBG), photonic band-gap materials, and photonic crystals (PCs) are important investigations [1,2]. The basic feature of PCs is the presence of permitted and forbidden frequency bands for light. It is possible to manipulate the light with PCs. Due to this property, PCs hold a great potential for designing new optical devices. There has been an increase in researches of tuning the optical properties of photonic band gap to design devices. Some tunable photonic band gap researches have been done in one-dimensional (1D) [3] and two dimensional (2D) [4-11] and 3D [12] PCs.

Materials are very important to determine the optical properties of a PC. The properties of PCs made of anisotropic materials different from those of isotropic PCs. Zabel and Stroud have been reported that the anisotropy of materials can split degenerate bands and this will close band gap of the PC [13]. Li et all. haveproved that the band gap can be increased by using the anisotropic materials in a PC [14].B. Rezaei and M. Kalafianalyzed the tunability of full band gap of anisotropic tellurium rods infiltratedwith LCs in air background [15] and 2D hexagonal PC of circular rods consisting of an inner rod and anisotropic outer shell aligned in a uniform background [16].B. Rezaeiet. all. are studied the absolute band gap properties of 2D PCs created by square, triangular and honeycomblattices of air holes in anisotropic tellurium background by changingthe shape and orientation of the holes [17]. Liu showed that the absolute bandgaps can be continuously tuned in the square and triangular lattices consisting of anisotropic-dielectric cylinders by infiltrating nematicLC [18].Liu et. all. are theoretically demonstrated the tunable bandgaps in 3D anisotropic PC structure with nematic LCs [19].Pan et. all. calculatedabsolute photonic band gaps for oval hollow anisotropic tellurium (Te) rods and square hollow anisotropic Te rods for the triangular and the square lattice [20]. 
In present paper, we theoretically demonstrated and developed the band gaps and optical properties in 2D anisotropic PC structure with nematic LCs. PC structure and materialswereespecially selected that optical properties are changed by external effect such as an electric field, magnetic field, light, temperature etc. The investigation is achieved by controlling intensity of band gap and optical properties added different material to a certain structure. The band gap is manipulated by rotating directors of LCs under the impact of an applied electric field. It was solved Maxwell's equations for the propagation of electromagnetic waves in a periodic arrangement of anisotropic PC with nematic LCs. Using the PWE method and FDTD method, the PC structure composed of an anisotropic LC in semiconductor square hollow nanorod is designed.

\section{Numerical Method}

The fundamentals of the PWE method and the FDTD method are based on a direct numerical solution of the time-dependent Maxwell's equations as illustrated in some articles [21-26].Generally LCs possess two kinds of dielectric constants. One is ordinary dielectric constants $\varepsilon_{o}$, and the other is extraordinary dielectric constants $\varepsilon_{e}$. Light waves with electric fields perpendicular and parallel to the director of the LC have ordinary and extraordinary refractive indices, respectively. The components of the dielectric tensor of the nematic LC are represented as [27]

$$
\begin{gathered}
\varepsilon_{x x}(\vec{r})=n_{o}^{2}+\left(n_{e}^{2}-n_{o}^{2}\right) \cos ^{2} \theta \cos ^{2} \emptyset \\
\varepsilon_{x y}(\vec{r})=\varepsilon_{y x}(\vec{r})=\left(n_{e}^{2}-n_{o}^{2}\right) \cos ^{2} \theta \sin \emptyset \cos \emptyset \\
\varepsilon_{x z}(\vec{r})=\varepsilon_{z x}(\vec{r})=\left(n_{e}^{2}-n_{o}^{2}\right) \sin \theta \cos \theta \cos \emptyset \\
\varepsilon_{y y}(\vec{r})=n_{o}^{2}+\left(n_{e}^{2}-n_{o}^{2}\right) \cos ^{2} \theta \sin ^{2} \emptyset \\
\varepsilon_{y z}(\vec{r})=\varepsilon_{z y}(\vec{r})=\left(n_{e}^{2}-n_{o}^{2}\right) \sin \theta \cos \theta \sin \emptyset \\
\varepsilon_{z z}(\vec{r})=n_{o}^{2}+\left(n_{e}^{2}-n_{o}^{2}\right) \sin ^{2} \theta
\end{gathered}
$$

where $\theta$ is the tilt angle of the LC director(i.e. the angle between the LC director and the XY-plane) and $\varnothing$ is the rotation angle between the projection of the LC director on the XY-plane and the Xaxis, and $\vec{n}$ is the director of the LC.

On the other hand Bloch's theorem [28] is used to expand the $H(\vec{r})$ field in terms of plane waves since the light waves are transmitted in periodic structures, as

$$
H(\vec{r})=\sum_{\vec{G}} h(\vec{G}) \hat{e}_{\vec{G}} e^{i(\vec{k}+\vec{G}) \cdot \vec{r}}
$$

where $\vec{k}$ is a wave vector in the Brillouin zone of the latticeand $\hat{e}_{\vec{G}}$ is the direction which is perpendicular tothe wave vector $(\vec{k}+\vec{G})$ owing to the transverse character ofthe magnetic field $H(\vec{r})$, $\nabla \cdot H(\vec{r})=0$.

\section{D Anisotropic PCs with LCs}

A novel type of two-dimensional anisotropic PC structure composed of an anisotropic nematicLC in semiconductor square hollow nanorod with lattice constant $a=1 \mu \mathrm{m}$ is presented. The PC structures are depicted in Fig. 1. It has considered (a) anisotropic Tenanorod, (b) hollow anisotropic Tenanorod, and (c) hollow anisotropic Tenanorodof nematic LC-infilled in air background $\left(\varepsilon_{a}=1\right)$ for square lattice. Anisotropic Tenanorodhas two different principle refractive indices as ordinaryrefractive index $n_{o}=4.8$ and extraordinary refractive index $n_{e}=6.2$. The ordinary and extraordinary refractive index of 5CB type (4-pentyl-4 -cyanobiphenyl) LCs are $n_{o}=1.548$ and $n_{e}=1.742$, respectively. Parameters $d_{1}$ and $d_{2}$ denote the inner and outer length of anisotropic Tenanorods, respectively.

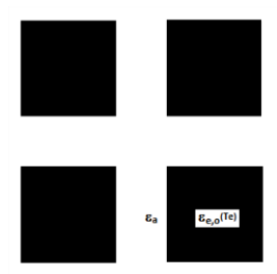

(a)

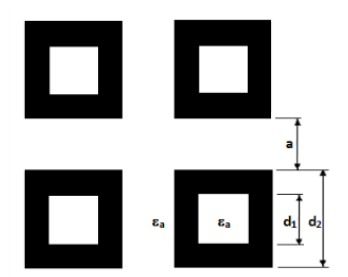

(b)

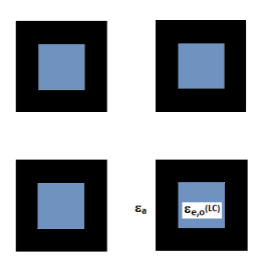

(c)

Fig. 1.For square lattice (a) anisotropic Tenanorod, (b) hollow anisotropic Tenanorod,and (c) hollow anisotropic Tenanorodof nematicLC-infiltrated in airbackground. 
Firstly, it is considered photonic band structure for square lattice of anisotropic solid Tenanorodin air background. It is assumed that $d_{2}=0.6 a$, the sides of square nanorodparallel to the primitive reciprocal lattice vectors. The PBG map for TE and TM mode which is calculated along to the high symmetry point for Brillouin zone in square lattice is plotted in Fig. 2.Relative width and center normalized frequency values are seen that two band gaps for TE modes and three band gaps for TM modes as shown in Table 1.Solving for TE band polarizationit is founded TE1 band gap value from band 1 to band 2, TE2 value from band 3 to band 4. Similarly, TM1 gap sizeis $19.6 \%$ between band 1 and band 2, TM2 is $13.3 \%$ between band 3 and band 4, and TM3 is $8.5 \%$ between band 6 and band 7 for TM band polarization.

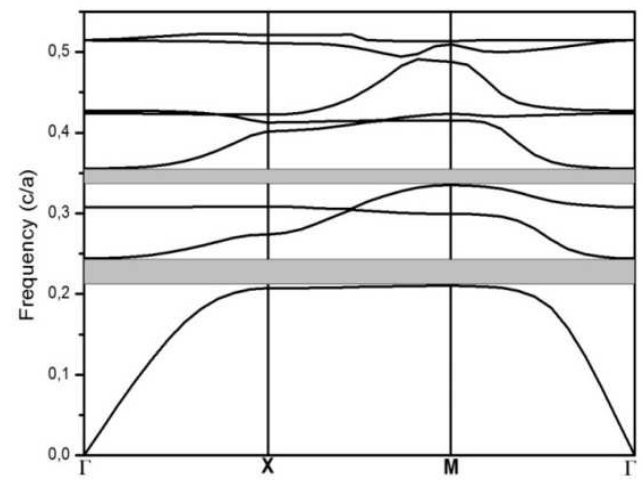

(a)

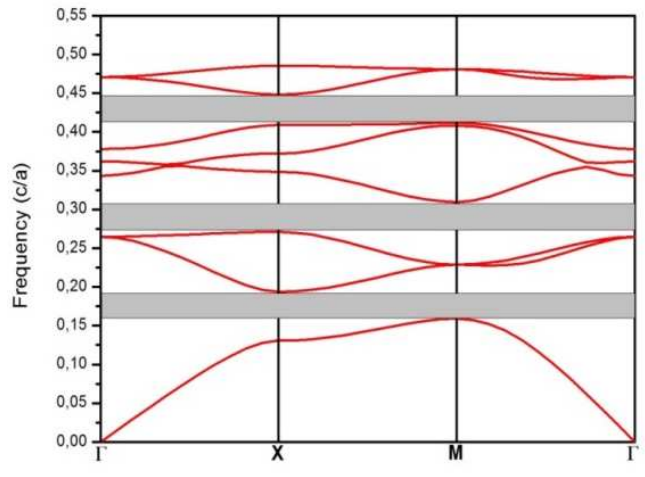

(b)

Fig. 2.Photonic band gap for (a) TE and (b) TM mode in square lattice of anisotropic Tenanorod in air background.

Table 1.Photonic band gap and gap size \% of TE and TM modes for square lattice of anisotropic Tenanorod in air background

\begin{tabular}{|c|c|c|c|c|c|}
\hline \multicolumn{3}{|c|}{ TM } & \multicolumn{3}{|c|}{ TE } \\
\hline & BandGap $[\omega \mathrm{a} / 2 \pi \mathrm{c}]$ & $\begin{array}{c}\text { Gap Size } \\
\%\end{array}$ & & $\begin{array}{l}\text { BandGap } \\
{[\omega \mathrm{a} / 2 \pi \mathrm{c}]}\end{array}$ & $\begin{array}{c}\text { Gap } \\
\text { Size } \\
\%\end{array}$ \\
\hline TM1 & $(0.159-0.193)$ & 19.6 & TE1 & $\begin{array}{c}(0.210- \\
0.244)\end{array}$ & 14.8 \\
\hline TM2 & $(0.271-0.309)$ & 13.3 & TE2 & $\begin{array}{c}(0.335- \\
0.356)\end{array}$ & 5.9 \\
\hline TM3 & $(0.411-0.448)$ & 8.5 & & & \\
\hline
\end{tabular}

Secondly, photonic band structure of hollow anisotropic Tenanorod in air background is considered. It is assumed that $d_{1}=0.3 a$ and $d_{2}=0.6 a$ denote the inner and outer length of hollow anisotropic Tenanorods.The band diagram for the second pattern is plotted in Fig. 3. When anisotropic Tenanorodis compared with hollow anisotropic Tenanorod,it is seen that variation of band gapnumber for TE mode is decreased and for TM mode is increased. Table 2 shows relative width and center normalized frequency values of hollow anisotropic Tenanorod in air background.

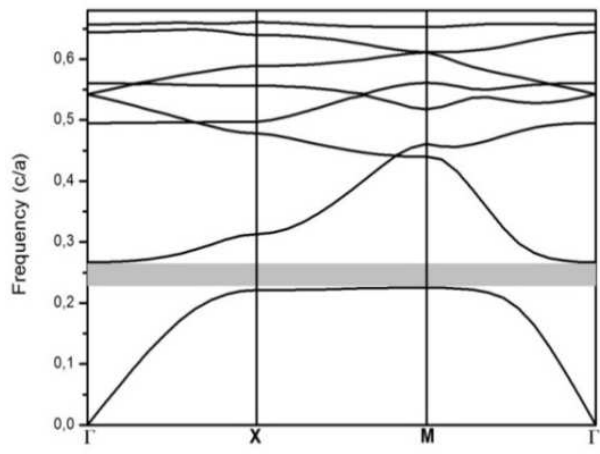

(a)

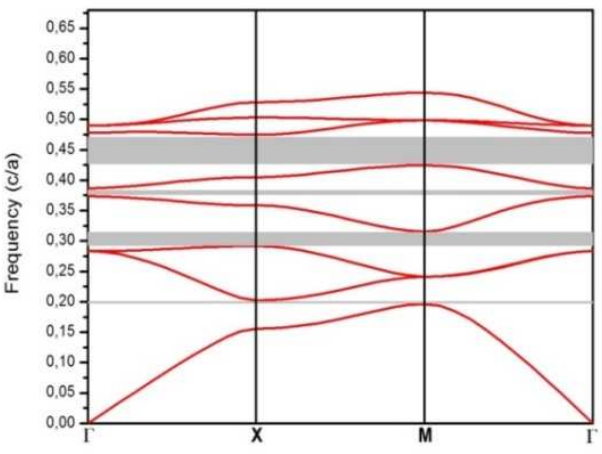

(b)

Fig. 3.Photonic band gap for (a) TE and (b) TM mode in square lattice of hollow anisotropic Tenanorod in air background. 
Table 2.Photonic band gap and gap size \% of TE and TM modes for square lattice of hollow anisotropic Tenanorod in air background

\begin{tabular}{|c|c|c|c|c|c|}
\hline \multicolumn{3}{|c|}{ TM } & \multicolumn{3}{|c|}{ TE } \\
\hline & BandGap $[\omega \mathrm{a} / 2 \pi \mathrm{c}]$ & $\begin{array}{c}\text { Gap Size } \\
\%\end{array}$ & & $\begin{array}{c}\text { BandGap } \\
{[\omega \mathrm{a} / 2 \pi \mathrm{c}]}\end{array}$ & $\begin{array}{c}\text { Gap Size } \\
\%\end{array}$ \\
\hline TM1 & $(0.196-0.202)$ & 2.9 & TE1 & $\begin{array}{c}(0.225- \\
0.267)\end{array}$ & 16.9 \\
\hline TM2 & $(0.291-0.315)$ & 7.9 & & & \\
\hline TM3 & $(0.373-0.386)$ & 3.5 & & & \\
\hline TM4 & $(0.424-0.474)$ & 11.2 & & & \\
\hline
\end{tabular}

Fig. 4 indicates thephotonic band structure of hollow anisotropic Tenanorod of nematic LCinfiltrated in air background for square lattice. For TE and TM modes band gap sizeof hollow anisotropic Tenanorod of nematic LC- infiltrated approximately is closed of hollow anisotropic Tenanorod as shown in Table 3. Compared hollow anisotropic Tenanorod of nematic LC-infiltrated in air background with hollow anisotropic Tenanorod in air background, it can be seen that band gap number increase in TE mode, and band gap number is the same in TM mode but band gap values are changed.

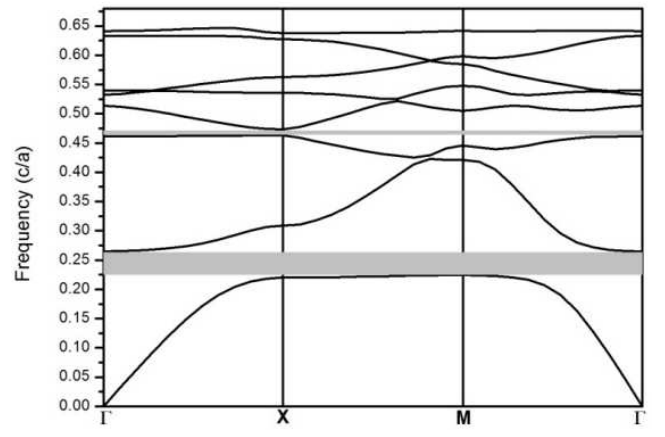

(a)

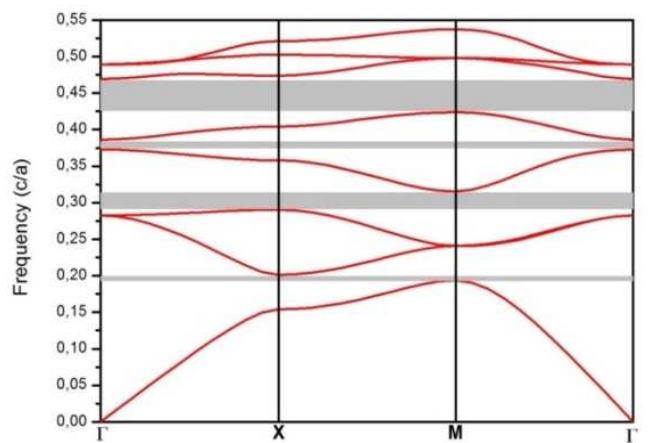

(b)

Fig. 4.Photonic band gap for (a) TE and (b) TM mode in square lattice of hollow anisotropic Tenanorod of nematic LC-infiltrated in air background.

Table 3. Photonic band gap and gap size \% of TE and TM modes for square lattice of hollow anisotropic Tenanorodof nematic LC-infiltrated in air background

\begin{tabular}{ccc|ccc}
\hline \hline & TM & \multicolumn{3}{|c}{ TE } \\
\hline TM1 & $(0.193-0.201)$ & 4.0 & TE1 & $\begin{array}{c}(0.224- \\
0.265)\end{array}$ & 16.8 \\
& & & & $\begin{array}{c}\text { BandGap } \\
\%\end{array}$ & $\begin{array}{c}\text { Gap Size } \\
\%\end{array}$ \\
TM2 & $(0.290-0.315)$ & 8.2 & TE2 & $0.473)$ & 2.2 \\
TM3 & $(0.372-0.386)$ & 3.5 & & & \\
TM4 & $(0.423-0.469)$ & 10.2 & & & \\
\hline \hline
\end{tabular}

It is well known that anisotropic nanostructuring PBG structure array are capable of changing the polarization state of transmitted or reflected light. Therefore we also calculated optical response of PBG structure for different rotation angle of the polarization ellipse and ellipticity of light transmitted through PBG array. Thenumerical results of variation of full band gap by changing the director of LC for photonic crystal structure rotated fully from $0^{\circ}$ to $90^{\circ}$ presents in Tables 4-5 and Fig. 5.Variation of band gap size $\%$ as a function of $\theta$ is demonstratedfor TE mode and TM mode in square lattice of hollow anisotropic Tenanorod of nematic LC-infiltrated in air backgroundshow that TE mode is not affected excessively from rotated photonic crystal structure (Fig. 5 (a)). The most widely gap affectingTE band structures arebetween $30^{\circ}$ and $60^{\circ}$. Particularly, while TE5 band gap is reached maximum value, TE2, TE4 and TE6 band gaps are closedat $\theta=45^{\circ}$. On the other hand, Fig. 5 (b) is indicated thatTM band structures are moredelicateto rotate angleof photoniccrystal struc- 
ture. The most important pointat $\theta=45^{\circ}$ unlike TE band gaps, TM band gaps reach to the minimum value. Furthermore, TM1 band gap which has the lowest frequency in TM mode are not altered via rotate angle.

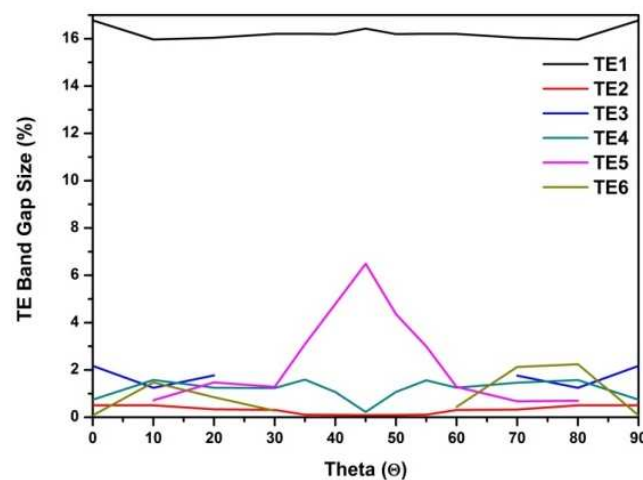

(a)

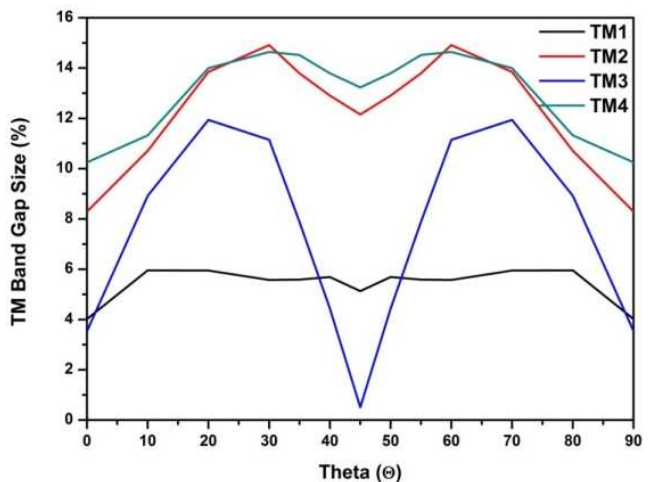

(b)

Fig. 5.Variation of bandgap size as a function of $\theta$ for (a) TE and (b) TM mode in square lattice of hollow anisotropic Tenanorod of nematic LC-infiltrated in air background.

Table 4.Variation of full band gap size for TE modes in square lattice of LC-infiltrated anisotropic tellurium nanorodin air background.

\begin{tabular}{|c|c|c|c|c|c|c|c|c|c|c|c|c|}
\hline \multicolumn{3}{|c|}{$\overline{\text { TE1 }}$} & \multicolumn{2}{|l|}{$\overline{\text { TE2 }}$} & \multicolumn{2}{|l|}{ TE3 } & \multicolumn{2}{|l|}{$\overline{\text { TE4 }}$} & \multicolumn{2}{|l|}{$\overline{\text { TE5 }}$} & \multicolumn{2}{|l|}{$\overline{\text { TE6 }}$} \\
\hline$\theta^{0}$ & $\begin{array}{l}\text { BandGap } \\
{[\omega \mathrm{a} / 2 \pi \mathrm{c}]}\end{array}$ & $\begin{array}{c}\text { Gap } \\
\text { Size \% }\end{array}$ & $\begin{array}{l}\text { BandGap } \\
{[\omega \mathrm{a} / 2 \pi \mathrm{c}]}\end{array}$ & $\begin{array}{c}\text { Gap } \\
\text { Size } \\
\% \\
\end{array}$ & $\begin{array}{l}\text { BandGap } \\
{[\omega \mathrm{a} / 2 \pi \mathrm{c}]}\end{array}$ & $\begin{array}{c}\text { Gap } \\
\text { Size } \\
\% \\
\end{array}$ & $\begin{array}{l}\text { BandGap } \\
{[\omega \mathrm{a} / 2 \pi \mathrm{c}]}\end{array}$ & $\begin{array}{c}\text { Gap } \\
\text { Size } \\
\% \\
\end{array}$ & $\begin{array}{l}\text { BandGap } \\
{[\omega \mathrm{a} / 2 \pi \mathrm{c}]}\end{array}$ & $\begin{array}{c}\text { Gap } \\
\text { Size } \\
\% \\
\end{array}$ & $\begin{array}{l}\text { BandGap } \\
{[\omega \mathrm{a} / 2 \pi \mathrm{c}]}\end{array}$ & $\begin{array}{c}\text { Gap } \\
\text { Size \% }\end{array}$ \\
\hline 0 & $\begin{array}{l}(0.224- \\
0.265)\end{array}$ & 16.77 & $(0.423-0.425)$ & 0.52 & $(0.463-0.473)$ & 2.17 & $(0.522-0.525)$ & 0.75 & $(0.590-0.591)$ & 0.07 & $(0.634-0.638)$ & 0.72 \\
\hline 10 & $\begin{array}{l}(0.231- \\
0.271)\end{array}$ & 15.97 & $(0.438-0.440)$ & 0.51 & $(0.481-0.487)$ & 1.25 & $(0.535-0.543)$ & 1.58 & $(0.567-0.571)$ & 0.72 & $(0.602-0.611)$ & 1.47 \\
\hline 20 & $\begin{array}{l}(0.231- \\
0.271)\end{array}$ & 16.04 & $(0.444-0.446)$ & 0.33 & $(0.487-0.496)$ & 1.77 & $(0.538-0.545)$ & 1.25 & $(0.567-0.576)$ & 1.47 & $(0.614-0.619)$ & 0.85 \\
\hline 30 & $\begin{array}{l}(0.229- \\
0.270)\end{array}$ & 16.21 & $(0.449-0.451)$ & 0.31 & - & - & $(0.534-0.540)$ & 1.23 & $(0.567-0.574)$ & 1.28 & $(0.621-0.623)$ & 0.28 \\
\hline 35 & $\begin{array}{l}(0.230- \\
0.271)\end{array}$ & 16.21 & $(0.454-0.455)$ & 0.10 & - & - & $(0.536-0.545)$ & 1.59 & $(0.569-0.586)$ & 3.08 & - & - \\
\hline 40 & $\begin{array}{l}(0.230- \\
0.271)\end{array}$ & 16.17 & $(0.454-0.455)$ & 0.09 & - & - & $(0.537-0.542)$ & 1.07 & $(0.565-0.593)$ & 4.79 & - & - \\
\hline 45 & $\begin{array}{l}(0.225- \\
0.266)\end{array}$ & 16.42 & $(0.445-0.446)$ & 0.09 & - & - & $(0.525-0.527)$ & 0.22 & $(0.549-0.586)$ & 6.49 & - & - \\
\hline 50 & $\begin{array}{l}(0.230- \\
0.271)\end{array}$ & 16.17 & $(0.454-0.455)$ & 0.09 & - & - & $(0.537-0.542)$ & 1.07 & $(0.565-0.591)$ & 4.36 & - & - \\
\hline 55 & $\begin{array}{l}(0.230- \\
0.271)\end{array}$ & 16.21 & $(0.454-0.455)$ & 0.10 & - & - & $(0.536-0.545)$ & 1.59 & $(0.569-0.586)$ & 3.08 & - & - \\
\hline 60 & $\begin{array}{l}(0.229- \\
0.270)\end{array}$ & 16.21 & $(0.449-0.451)$ & 0.31 & - & - & $(0.534-0.540)$ & 1.23 & $(0.567-0.574)$ & 1.28 & $(0.621-0.624)$ & 0.44 \\
\hline 70 & $\begin{array}{l}(0.231- \\
0.271)\end{array}$ & 16.04 & $(0.444-0.446)$ & 0.33 & $(0.487-0.496)$ & 1.77 & $(0.537-0.545)$ & 1.46 & $(0.569-0.572)$ & 0.68 & $(0.612-0.625)$ & 2.13 \\
\hline 80 & $\begin{array}{l}(0.231- \\
0.271)\end{array}$ & 15.97 & $(0.438-0.440)$ & 0.51 & $(0.481-0.487)$ & 1.25 & $(0.535-0.543)$ & 1.58 & $(0.567-0.571)$ & 0.72 & $(0.602-0.616)$ & 2.24 \\
\hline 90 & $\begin{array}{l}(0.224- \\
0.265)\end{array}$ & 16.77 & $(0.423-0.425)$ & 0.52 & $(0.463-0.473)$ & 2.17 & $(0.522-0.525)$ & 0.75 & $(0.590-0.591)$ & 0.07 & $(0.634-0.638)$ & 0.72 \\
\hline
\end{tabular}

Table 5.Variation of full band gap size for TM modes in square lattice of LC-infiltrated anisotropic tellurium nanorodin air background.

\begin{tabular}{|c|c|c|c|c|c|c|c|c|}
\hline \multicolumn{3}{|c|}{ TM1 } & \multicolumn{2}{|l|}{ TM2 } & \multicolumn{2}{|l|}{ TM3 } & \multicolumn{2}{|l|}{ TM4 } \\
\hline$\theta^{0}$ & $\operatorname{BandGap}[\omega \mathrm{a} / 2 \pi \mathrm{c}]$ & $\begin{array}{c}\text { Gap } \\
\text { Size } \%\end{array}$ & BandGap $[\omega \mathrm{a} / 2 \pi \mathrm{c}]$ & $\begin{array}{c}\text { Gap } \\
\text { Size } \%\end{array}$ & BandGap $[\omega \mathrm{a} / 2 \pi \mathrm{c}]$ & $\begin{array}{c}\text { Gap } \\
\text { Size } \%\end{array}$ & BandGap $[\omega \mathrm{a} / 2 \pi \mathrm{c}]$ & $\begin{array}{c}\text { Gap } \\
\text { Size \% }\end{array}$ \\
\hline 0 & $(0.193-0.201)$ & 4.02 & $(0.290-0.315)$ & 8.29 & $(0.372-0.386)$ & 3.54 & $(0.423-0.469)$ & 10.25 \\
\hline 10 & $(0.195-0.207)$ & 5.95 & $(0.295-0.328)$ & 10.71 & $(0.370-0.405)$ & 8.92 & $(0.431-0.483)$ & 11.32 \\
\hline 20 & $(0.196-0.208)$ & 5.94 & $(0.297-0.341)$ & 13.84 & $(0.367-0.414)$ & 11.94 & $(0.425-0.489)$ & 13.99 \\
\hline 30 & $(0.197-0.209)$ & 5.58 & $(0.297-0.345)$ & 14.91 & $(0.368-0.411)$ & 11.14 & $(0.423-0.490)$ & 14.64 \\
\hline 35 & $(0.197-0.209)$ & 5.58 & $(0.298-0.343)$ & 13.79 & $(0.374-0.405)$ & 7.88 & $(0.424-0.490)$ & 14.52 \\
\hline
\end{tabular}




\begin{tabular}{lll|ll|ll|ll}
40 & $(0.197-0.208)$ & 5.69 & $(0.299-0.340)$ & 12.90 & $(0.381-0.398)$ & 4.43 & $(0.426-0.489)$ & 13.79 \\
45 & $(0.195-0.205)$ & 5.13 & $(0.295-0.334)$ & 12.15 & $(0.384-0.386)$ & 0.51 & $(0.421-0.480)$ & 13.23 \\
50 & $(0.197-0.208)$ & 5.69 & $(0.299-0.340)$ & 12.90 & $(0.381-0.398)$ & 4.43 & $(0.426-0.489)$ & 13.79 \\
55 & $(0.198-0.208)$ & 5.58 & $(0.298-0.343)$ & 13.79 & $(0.374-0.405)$ & 7.88 & $(0.424-0.490)$ & 14.52 \\
60 & $(0.197-0.209)$ & 5.58 & $(0.297-0.208)$ & 14.91 & $(0.368-0.411)$ & 11.14 & $(0.423-0.490)$ & 14.64 \\
70 & $(0.196-0.208)$ & 5.94 & $(0.297-0.341)$ & 13.84 & $(0.367-0.414)$ & 11.94 & $(0.425-0.489)$ & 13.99 \\
80 & $(0.195-0.207)$ & 5.95 & $(0.295-0.328)$ & 10.71 & $(0.370-0.405)$ & 8.92 & $(0.431-0.483)$ & 11.32 \\
90 & $(0.193-0.201)$ & 4.02 & $(0.290-0.315)$ & 8.29 & $(0.372-0.386)$ & 3.54 & $(0.423-0.469)$ & 10.25 \\
\hline \hline
\end{tabular}

Fig. 6 is shown as variation of TE bandgap size and TM bandgap size as a function of $n_{e}$ and $n_{o}$ forsquarelattice of hollow anisotropic Tenanorod of nematic LC-infiltrated in air background. As seen in Fig. 6 (a) there exist two photonic band gaps in TE band structure. It is clear that TE1 band gap size is more sensitive than TE2 band gap size to anisotropic refractive index. TE2 band gap quite is low for $n_{e}<4$ and $n_{o}<2$. Moreover, the variation of $n_{e}$ is more influenced TE1 band gap than $n_{o}$. On the other hand, TE1 band gap is close to zero for $n_{e} \leq 4$ and $n_{o} \leq 4$. It can be seen from Fig. 6 (b) that TM band structure ofsquarelattice of hollow anisotropic Tenanorod of nematic LCinfiltrated have fully four band gaps (TM1, TM2, TM3 and TM4). The variation of this band gaps according to anisotropic refractive index are similar to each other. In contrast TE band gap size, it is seen in Fig. 6 (b) that TM band gap size is linearly depended on anisotropic refractive index.

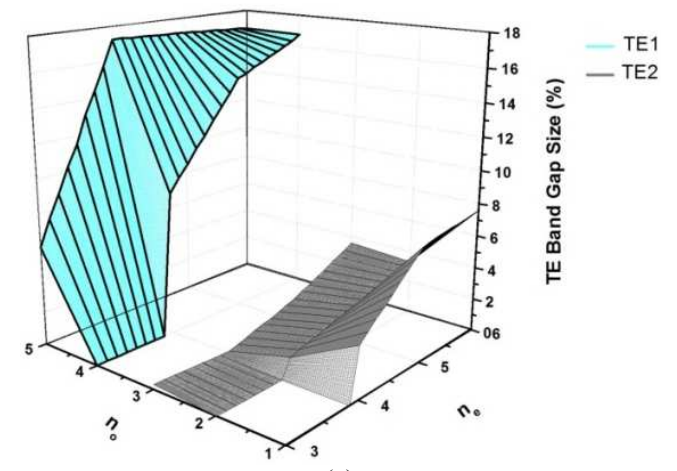

(a)

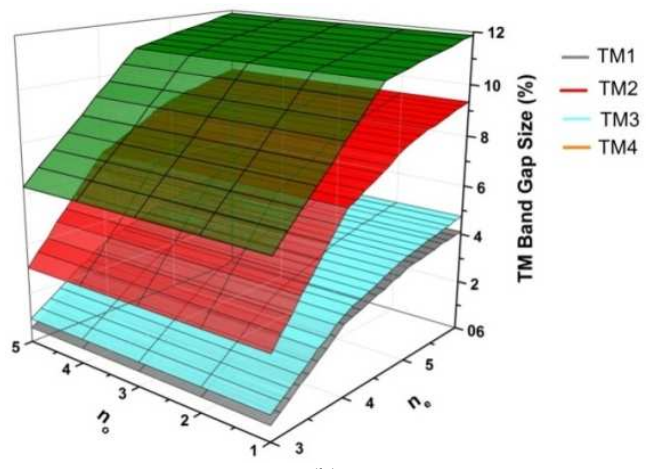

(b)

Fig. 6.Variation of TE band gap size and TM band gap size as a function of $n_{e}$ and $n_{o}$ for square lattice of hollow anisotropic Tenanorod of nematic LC-infiltrated in air background.

\section{Conclusion}

It was theoretically studied the optical properties in 2D anisotropic PC structure modulated bynematic LCs. The investigation was achieved by selecting convenient structure and materials. The PC structure consisted of an anisotropic nematicLC (5CB) in semiconductor (Te) square hollow nanorod is designed using the PWE method and the FDTD method.Numerical results were shown that optical properties of the photonic structure can be controlled by rotating directors of LC. When photonic crystal structure rotated fully from $0^{\circ}$ to $90^{\circ}$, absolute band gap existed between $40^{\circ}$ and $50^{\circ}$.

\section{References}

[1] E. Yablonovitch, J. Opt. Soc. Am. B Vol. 10 (1993), p. 283.

[2] J.N. Winn, R.D. Meade, J.D. Joannopoulos, J. Mod. Optics Vol. 41 (1994), p. 257.

[3] G. Alagappan, X. W. Sun, P. Shum, M. B. Yu, M. T. Doan, J. Opt. Soc. Am. B Vol. 23 (2006), p. 159.

[4] C.S. Kee, H. Lim, Phys. Rev.B Vol. 64 (2001), p. 121103-1.

[5] S.M. Hsu, M.M. Chen, H.C. Chang, Opt. Express Vol. 15 (2007), p. 5416.

[6] C. Schuller, F. Klopf, J.P. Reithmaier, M. Kamp, A. Forchel, Appl. Phys. Lett. Vol. 82 (2003), p. 2767. 
[7] D. Liu, Y. Gao, D. Gao, X. Han, Optics Commun. Vol. 285 (2012), p. 1988.

[8] N. Zhu, J. Wang, C. Cheng, X. Yan, Optik Vol. 124 (2013), p. 309.

[9] Z.Y. Li, B.Y. Gu, G.Z. Yang, Phy. Rev. Lett. Vol. 81 (1998), p. 2574.

[10] H. Takeda, K. Yoshino, Phy. Lett. E Vol. 70 (2004), p. 026601-1.

[11] G. Alagappan, X. W. Sun, P. Shum, M. B. Yu, D. den Engelsen, J. Opt. Soc. Am. A Vol. 23 (2006), p. 2002.

[12] K. M. Ho, C. T. Chan, C. M. Soukoulis, Phys. Rev. Lett. Vol. 65 (1990), p. 3152.

[13] I. H. H. Zabel, D. Stroud, Phys. Rev. B Vol. 48 (1993), p. 5004.

[14] Z. Y. Li, J. Wang, B.Y. Gu, Phys. Rev. B Vol. 58 (1998), p. 3721.

[15] B. Rezaei, M. Kalafi, Optics Commun. Vol. 282 (2009), p. 1584.

[16] B. Rezaei, M. Kalafi, Optics Commun. Vol. 266 (2006), p. 159.

[17] B. Rezaei, T. F. Khalkhali, A. S. Vala, M. Kalafi, Optics Commun. Vol. 282 (2009), p. 2861.

[18] C.-Y. Liu, Phy. Lett. A Vol. 372 (2008), p. 5198.

[19] C.-Y. Liu, Y.-T.Peng, J.-Z.Wang, L.-W. Chen, Physica B Vol. 388 (2007), p. 124.

[20] T. Pan, F. Zhuang, Z. Y. Li, Solid State Commun. Vol. 129 (2004), p. 501.

[21] C.Y. Liu, L.W. Chen, IEEE Photonics Techn. Lett. Vol. 16 (2004), p. 1849.

[22] C.Y. Liu, L.W. Chen, J. of Nanomaterials Vol. 2006 (2006), p. 1.

[23] A. Taflove, in: Advances in Computational Electrodynamics: The Finite-Difference TimeDomain Method, chapter, 1, Artech House, Boston, Mass, USA, (1998).

[24] Z. Ma, K. Ogusu, , Optics Commun. Vol. 282 (2009), p. 1322.

[25] M. Plihal, A. A. Maradudin, Phys. Rev. B Vol. 44 (1991), p. 8565.

[26] J.D. Joannopoulos, R.D. Meade, J.N. Winn, in: Photonic Crystals: Molding the Flow of Light, chapter, 2, second ed., Princeton University Press, Princeton, NJ, (1995).

[27] I.-C. Khoo, S.-T. Wu, in: Optics and Nonlinear Optics of Liquid Crystals, chapter, 2, World Scientific, Singapore, (1993).

[28]Kittel, Charles, in:Introduction to Solid State Physics,chapter, 7,Wiley,New York,(1996). 
Mechatronics, Applied Mechanics and Energy Engineering

10.4028/www.scientific.net/AMM.394

2D Anisotropic Photonic Crystals of Hollow Semiconductor Nanorod with Liquid Crystals 10.4028/www.scientific.net/AMM.394.38 